\title{
FINITE EXTENSIONS OF RINGS
}

\author{
BARBARA CORTZEN AND LANCE W. SMALL
}

(Communicated by Donald S. Passman)

\begin{abstract}
The paper concerns some cases of ring extensions $R \subset S$, where $S$ is finitely generated as a right $R$-module and $R$ is right Noetherian. In $\S 1$ it is shown that if $R$ is a Jacobson ring, then so is $S$, with the converse true in the PI case. In $\S 2$ we show that if $S$ is semiprime PI, $R$ must also be left (as well as right) Noetherian and $S$ is finitely generated as a left $R$-module. $\S 3$ contains a result on $E$-rings.
\end{abstract}

In this paper we collect some results concerning the relationship between rings $R \subset S$, where $S$ is finitely generated as a right $R$-module. For the special cases in which the finite extension $S$ of $R$ is normalizing or centralizing, many theorems have been proved. In this paper we obtain some results of a more general nature.

1. Jacobson property. We call a ring $R$ a Jacobson ring if every prime ideal of $R$ is an intersection of primitive ideals. Let $J(R)$ denote the Jacobson radical of $R$ and $N(R)$ the lower nilradical, that is, the intersection of all the prime ideals of $R$. With this notation, the alternative formulations of the Jacobson property are:

$J(R / P)=0$ for all prime ideals $P$ of $R$.

$J(\bar{R})=N(\bar{R})$ for every homomorphic image $\bar{R}$ of $R$.

If $R$ is PI or right Noetherian, then every nil ideal is contained in $N(R)$, and the last condition is equivalent to:

$J(\bar{R})$ is nil for every homomorphic image $\bar{R}$ of $R$.

THEOREM 1. Let $R$ be a right Noetherian subring of a ring $S$, such that $S$ is finitely generated as a right $R$-module. Then, if $R$ is Jacobson, so must $S$ be Jacobson.

ProOF. Given any prime ideal $P$ of $S$, we want to show that $J(S / P)=0$. Since the hypothesis of the theorem holds for the ring embedding $R / P \cap R \subset S / P$, the problem reduces to the case where $S$ is a prime ring. We therefore want to show that, if $S$ is prime, then $J(S)=0$.

Suppose $J(S) \neq 0$. By a standard result on Goldie rings, $J(S)$ must contain a regular element, say $a$. Since $S_{R}$ is finitely generated and $R$ is right Noetherian, there is a positive integer $n$, such that the elements $1, a, a^{2}, \ldots, a^{n}$ are integrally dependent over $R$. That is, for some $r_{n-1}, \ldots, r_{1}, r_{0} \in R$, we have $a^{n}+a^{n-1} r_{n-1}+$ $\cdots+a r_{1}+r_{0}=0$. If $n$ is minimal, then, since $a$ is regular, $r_{0} \neq 0$. But then $r_{0} \in J(S) \cap R$. Hence $J(S) \cap R \neq 0$.

We claim that $J(S) \cap R \subset J(R)$. Let $x \in J(S) \cap R$ and suppose $x \notin J(R)$. Then there is an element $r \in R$ for which $1-r x$ is not invertible in $R$. But $1-r x$ is

Received by the editors March 31, 1987.

1980 Mathematics Subject Classification (1985 Revision). Primary 16A38, 16A33, 16A21. 
invertible in $S$, say $(1-r x) s=1$ for some $s \in S$. Since $s$ is integral over $R$ we have $s^{k}+s^{k-1} a_{k-1}+\cdots+s a_{1}+a_{0}=0$ for some $a_{0}, a_{1}, \ldots, a_{k-1} \in R$. Multiplying this equation by $(1-r x)^{k-1}$ and solving for $s$ yields:

$$
s=-\left[a_{k-1}+(1-r x) a_{k-2}+\cdots+(1-r x)^{k-1} a_{0}\right] \in R,
$$

which is a contradiction. Consequently, $J(S) \cap R \subset J(R)$.

Because $R$ is right Noetherian and Jacobson, we have that $J(R)=N(R)$, the maximal nilpotent ideal of $R$. Hence $J(S) \cap R$ is a nonzero nilpotent ideal of $R$.

Consider the ring embedding $R / J(S) \cap R \subset S / J(S)$. This is a finite ring extension. Invoking some results on Krull dimension (as defined in [6]), we shall obtain a contradiction. We use notation $|R|$ for Krull dimension of the ring $R$, and $|M|_{R}$ for Krull dimension of the $R$-module $M$.

On one hand, since $J(S) \neq 0$ and $S$ is prime, $J(S)$ contains a regular element and therefore $|S / J(S)|_{R}<|S|_{R}=|R|$.

On the other hand, since $J(S) \cap R$ is nilpotent, we have:

$$
|R|=|R / J(S) \cap R|=|S / J(S)|_{R / J(S) \cap R}=|S / J(S)|_{R},
$$

which contradicts the inequality above.

The proof is now complete, since the assumption $J(S) \neq 0$ led to a contradiction.

In the PI case, the converse of Theorem 1 holds. To prove this, note that the Jacobson property goes up and down for the following two classes of ring extensions. First, if $S$ is a liberal extension of $R$, that is, if $S$ is finitely generated as a module over $R$ by elements that centralize $R$, then $S$ is Jacobson if and only if $R$ is Jacobson (Robson and Small [8]). The second type of extensions that we need is considered in the following lemma, half of which follows immediately from Blair's results in $[3]$.

LEMMA. Suppose $R$ is a central subring of a PI ring $S$ and that $S$ is integral over $R$. Then $S$ is Jacobson if and only if $R$ is Jacobson.

PROOF. Blair [3] proved that, if $S$ is integral over its subring $R$, for every prime ideal $P$ of $R$, there is a prime ideal $Q$ of $S$ with $Q \cap R=P$. Moreover, $J(R)=J(S) \cap R$.

If $S$ is a Jacobson ring, let $P$ be a prime ideal of $R$ and $Q$ a prime of $S$ lying over $P$. The hypothesis of the lemma carries over to the ring extension $R / P \subset S / Q$ and we have $J(R / P)=J(S / Q) \cap R / P=0$. Since $P$ was an arbitrary prime ideal of $R, R$ is Jacobson. This part of the lemma did not require the hypothesis that $S$ be PI.

Conversely, let $R$ be Jacobson. To show that $S$ is Jacobson, it is enough to prove that $J(S / Q)$ is nil for every ideal $Q$ of $S$. Since the hypothesis of the lemma holds for the ring embedding $R / Q \cap R \subset S / Q$, it is enough to show that if $R$ is Jacobson, then $J(S)$ is nil. Let $x \in J(S) . R\langle x\rangle$, the ring generated by $R$ and $x$, is a Jacobson ring, since it is a homomorphic image of the polynomial ring $R[X]$. As in the proof of Theorem 1, integrality of $R\langle x\rangle$ over $R$ gives $J(S) \cap R\langle x\rangle \subset J(R\langle x\rangle)$. Consequently, $x \in J(R\langle x\rangle)$ and $x$ is nilpotent. Thus $J(S)$ is nil. 
THEOREM 2. Let $R \subset S$ be rings such that $R$ is right Noetherian, $S$ is $P I$ and finitely generated as a right $R$-module. Then $S$ is Jacobson if and only if $R$ is Jacobson.

ProOF. In view of Theorem 1 , it is enough to show that " $S$ Jacobson" implies " $R$ Jacobson".

If $P$ is a prime ideal of $R$, there is a prime ideal $P^{\prime}$ of $S$ for which $P^{\prime} \cap R \subset P$ (take $P^{\prime}$ to be maximal among all ideals $I$ of $S$ for with $I \cap R \subset P$ ). Now consider the ring embedding of $R / P^{\prime} \cap R$ in $S / P^{\prime}$. If $R / P^{\prime} \cap R$ is Jacobson, then so is $R / P$. Thus one can replace $S$ by $S / P^{\prime}$ and assume that $S$ is prime.

By Posner's theorem, $S$ has a classical ring of quotients $Q$, which is a finitedimensional central simple algebra, $Q=M_{n}(D)$, where $D$ is a division ring finitedimensional over its center. If $Z$ is the center of $D$ and $K$ a maximal subfield of $D$, then we have $S \subset Q \subset M_{n}(D) \otimes_{z} K=M_{m}(K)$ for some $m$. Thus every element of $S$ can be considered as a matrix and has a (reduced) characteristic polynomial with coefficients in $Z$. Let $T$ denote the subalgebra of $M_{m}(K)$ generated by coefficients of these polynomials and let $T S$ denote the subalgebra of $M_{n}(D)$ generated by $T$ and $S, T R$ the subalgebra generated by $T$ and $R$. (For the construction and properties of the reduced trace ring $T S$, see [10 and $\mathbf{1}]$.)

Consider the diagram

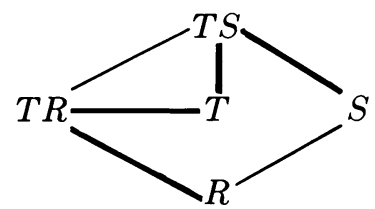

The path we take in proving the implication " $S$ Jacobson $\Rightarrow R$ Jacobson" is indicated by bold line segments. Note that all rings in the diagram are PI.

It is well known that, if $S$ is right Notherian, then $T S$ is a finite right $S$-module [1]. By Theorem 1, TS is Jacobson. It is also well known that $T S$ is integral over its center [1], and therefore also over $T$.

By the lemma above, $T$ must be Jacobson. $T R$ is integral over $T$ (as a subring of $T S$ ), and so is Jacobson. Since $T S$ is a finite $S$-module, it is also finitely generated over $R$, as is its $R$-submodule $T R$. Thus $T R$ is a liberal extension of $R$, and consequently $R$ is Jacobson. This completes the proof.

2. Changing sides. We shall be concerned now with some situations in which a right-hand property of a ring can be transferred to a left-hand one. The results we obtain will be of a going-down type. We cite two results which will be used in the proofs. The first of these results was proved by Cauchon [4] for the prime case. To extend this to the semiprime case is routine (see $[\mathbf{9}, \mathrm{pp} .225-226])$. The result is

(i) A semiprime PI ring which satisfies ACC on ideals is right (and left) Noetherian.

The second result is due to Björk [2].

(ii) If $R$ is a right Noetherian subring of a right artinian ring $S$ such that $S$ is finitely generated as a right $R$-module, then $R$ is right artinian. 
THEOREM 3. Let $S$ be a semiprime $P I$ ring and $R$ a right Noetherian subring of $S$ such that $S$ is a finitely generated right $R$-module. Then $S$ is finitely generated as a left $R$-module and $R$ is left Noetherian.

ProOF. Consider the inclusion of rings: $R[x] \subset R+x S[x] \subset S[x]$. Since $S_{R}$ is finitely generated, $S$ is right Noetherian. Therefore $R[x]$ and $S[x]$ are also right Noetherian, as is the finitely generated $R[x]$-module $R+x S[x]$. Since $S$ has no nonzero nil ideals, $S[x]$ is semiprime. Because $S[x]$ is a PI ring, so is its subring $R+x S[x] . R+x S[x]$ is also semiprime, since it contains an ideal of a semiprime ring.

By the cited result (i) above, $R+x S[x]$ is left (as well as right) Noetherian. But then $R$, as a homomorphic image of $R+x S[x]$ must also be Noetherian.

The ideal $x S[x]$ of $R+x S[x]$ is finitely generated on the left, since $R+x S[x]$ is left Noetherian. Its homomorphic image $x S[x] / x^{2} S[x]$ has the same $R+x S[x]$ module structure as the $R$-module structure of $S$. Thus $S$ is a finitely generated left $R$-module.

COROLlaRY. Let $S$ be a primitive PI ring and $R$ a right Noetherian subring of $S$ such that $S$ is a finitely generated right $R$-module. Then $R$ is left and right artinian and $S$ is finitely generated as a left $R$-module.

ProOF. By a famous theorem of Kaplansky, $S$ is a simple algebra, finitedimensional over its center. By Theorem $3, R$ is both left and right Noetherian and $S$ is a finitely generated left $R$-module. By the cited result (ii) of Björk we get that $R$ is left and right artinian.

The following example shows that the condition that $S$ be a PI ring is necessary.

P. M. Cohn [5] has constructed a pair of division rings $D^{\prime} \subset D$ such that $D$ is a finite-dimensional right vector space over $D^{\prime}$ but is infinite-dimensional as a left vector space over $D^{\prime}$.

Let

$$
R=\left[\begin{array}{cc}
D^{\prime} & D \\
0 & D
\end{array}\right] \text { and } S=\left[\begin{array}{cc}
D & D \\
D & D
\end{array}\right]
$$

Thus $S$ is a simple artinian ring and, as is easily checked, is finitely generated both as a left and right $R$-module. Moreover, $R$ is right Noetherian. However, $R$ is right artinian, but is not left artinian (nor left Noetherian).

3. $E$-rings. A ring $S$ has been defined to be an $E$-ring [7] if its primitive ideals are coartinian, that is, if $S / P$ is simple artinian for every primitive ideal $P$ of $S$. Examples of such $E$-rings are Noetherian PI rings and, more generally, all fully bounded Noetherian rings.

The following result is interesting in view of Stafford's example in [11] of prime Noetherian rings $R \subset S$ and a simple $S$-module which has an infinite length over $R$, even though $S$ is finitely generated both as a left and right $R$-module.

THEOREM 4. Let $S$ be an E-ring and $R$ a right Noetherian subring of $S$ such that $S$ is finitely generated as a right $R$-module. Then every simple right $S$-module has finite length as a right $R$-module. Hence every right $S$-module of finite length has finite length as a right $R$-module.

ProOF. Let $M$ be a simple right $S$-module and set $A=\operatorname{Ann}_{S} M$, the annihilator of $M$ in $S$. Then $A$ is a primitive ideal of $S$, and $R / A \cap R$ is a subring of the simple 
artinian ring $S / A$. Clearly $S / A$ is finitely generated as a right $R / A \cap R$-module. By Björk's result, $R / A \cap R$ is right artinian. Since $M$ is finitely generated over $R / A \cap R, M$ has finite length as an $R$-module.

\section{REFERENCES}

1. S. A. Amitsur and L. W. Small, Prime ideals in PI-rings, J. Algebra 62 (1980), 358-383.

2. J.-E. Björk, Noetherian and Artinian chain conditions of associative rings, Arch. Math. 24 (1973), 366-379.

3. W. D. Blair, Right Noetherian rings integral over their centers, J. Algebra 27 (1973), 187-198.

4. G. Cauchon, Anneaux premiers, Noetheriens, a identites polynomiales, Bull. Soc. Math. France 104 (1976), 99-111.

5. P. M. Cohn, Quadratic extensions of skew fields, Proc. London Math. Soc. 3 (1951), 531-556.

6. R. Gordon and J. C. Robson, Krull dimension, Mem. Amer. Math. Soc., no. 133, 1973.

7. I. N. Herstein and L. W. Small, An extension of a theorem of Schur, Linear and Multilinear Algebra 3 (1975), 41-43.

8. J. C. Robson and L. W. Small, Liberal extensions, Proc. London Math. Soc. 3(42) (1981), 87-103.

9. L. H. Rowen, Polynomial identities in ring theory, Academic Press, New York, 1980.

10. W. Schelter, Non-commutative affine P.I. rings are catenary, J. Algebra 51 (1978), $12-18$.

11. J. T. Stafford, Non-holonomic modules over Weyl algebras and enveloping algebras, Invent. Math. 79(3) (1985), 619-638.

Department of Mathematics, DePaul University, Chicago, Illinois 60614

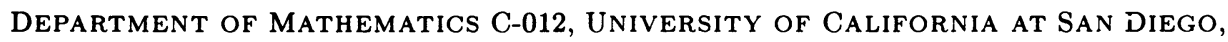
LA Jolla, CALifornia 92093 